\title{
The Effect of Low-pass Pre-filtering on Subvoxel Registration Algorithms in Digital Volume Correlation: A revisited study
}

\author{
Xiang Zou ${ }^{1}$, Kai Li ${ }^{2}$, Bing Pan ${ }^{1,3}$ \\ ${ }^{1}$ Institute of Solid Mechanics, Beihang University, Beijing 100191, China, zouxiang@buaa.edu.cn \\ ${ }^{2}$ School of Mechanics and Engineering Science, Shanghai University, Shanghai 200444, China \\ ${ }^{3}$ Beijing Advanced Discipline Center of Unmanned Aircraft System, Beihang University, Beijing 100191, China
}

\begin{abstract}
In digital volume correlation (DVC), random image noise in volumetric images leads to increased systematic error and random error in the displacements measured by subvoxel registration algorithms. Previous studies in DIC have shown that adopting low-pass pre-filtering to the images prior to the correlation analysis can effectively mitigate the systematic error associated with the classical forward additive NewtonRaphson (FA-NR) algorithm. However, the effect of low-pass pre-filtering on the state-of-the-art inverse compositional Gauss-Newton (ICGN) algorithm has not been investigated so far. In this work, we focus on the effect of low-pass pre-filtering on two mainstream subvoxel registration algorithms (i.e., 3D FA-NR algorithm and 3D IC-GN algorithm) used in DVC. Basic principles and theoretical error analyses of the two algorithms are described first. Then, based on numerical experiments with precisely controlled subvoxel displacements and noise levels, the influences of image noise on the displacements measured by two subvoxel algorithms are examined. Further, the effects of lowpass pre-filtering on these two subvoxel algorithms are examined for simulated image sets with different noise levels and deformation modes. The results show that the low-pass pre-filtering can effectively suppress the systematic errors for the 3D FA-NR algorithm, which is consistent with the previously drawn conclusion in DIC. On the contrary, different form the 3D FA-NR algorithm, the 3D IC-GN algorithm itself can reduce the influence of image noise, and the effect of low-pass pre-filtering on it is not so obvious as on 3D FA-NR algorithm.
\end{abstract}

Keywords: Digital volume correlation, subvoxel registration algorithm, image noise, image pre-filtering.

\section{INTRODUCTION}

Digital volume correlation (DVC) [1], [2], combined with advanced volumetric imaging facilities, has been established as a powerful technique for retrieving full-field internal deformation in opaque materials and biological tissues. Since it was first introduced by Bay [1] in 1999 to obtain the continuum-level displacement and strain fields in a trabecular bone, DVC has been increasingly used for characterizing the interior kinematic fields of various materials and structures [3]-[9]. The internal deformation in response to external loading obtained by DVC helps to understand the mechanical behaviors of materials and bridge the gap among theoretical prediction, numerical simulation, and experiments.

Like digital image correlation (DIC), the 2D counterpart of DVC [10], [11], the measurement accuracy and efficiency of DVC are dependent on the subvoxel registration algorithm. In the literature, different subpixel/subvoxel registration algorithms have been developed [1], [12], [13]. To find a better subpixel registration algorithm, Pan et al. [15] compared the performance of three commonly used registration algorithms (i.e., peak-finding algorithm, gradient-based algorithm, and Newton-Raphson (NR) algorithm), and found that the NR algorithm, an iterative spatial domain cross-correlation algorithm, is more accurate and stable. After that, to further improve the performance of the classic NR algorithm, continuous refinements have been made to reduce its complexity, improve its robustness and extend its applicability [16], [17]. The NR algorithm has also been extended to DVC for accurate subvoxel registration [18], [19]. Although being the most widely used and the most accurate algorithm for subpixel/subvoxel motion estimation, one significant drawback of the NR algorithm is its huge computational cost. To cope with this drawback, Pan et al. [20] proposed an advanced inverse-compositional GaussNewton (IC-GN) algorithm that can eliminate redundant calculations inherent in the NR algorithm. IC-GN algorithm is not only more computationally efficient than the NR algorithm without any loss of its measurement accuracy, but it also offers better noise-proof robustness performance [21], [22]. Due to these merits, the IC-GN algorithm has been considered as a gold standard and milestone algorithm for subpixel/subvoxel registration in practical DIC/DVC applications [23], [24].

In DIC/DVC, the systematic errors in measured displacements related to the subpixel/subvoxel registration algorithm can be classified into bias error associated with 
undermatched shape functions [25], imperfect interpolation and random noise [26]-[29]. To mitigate the systematic errors arisen from the use of undermatched shape functions, several effective approaches, including a sophisticated self-adaptive DVC approach that can adaptively determine the optimal subvolume size and shape functions at each calculation point, and quasi-Gauss DIC/DVC method [32], have been proposed [30], [31]. Also, to decrease the interpolation bias error and noise-induced bias error, a simple and effective approach applying a low-pass prefiltering prior to correlation analysis, was also presented. It is proved that the low-pass pre-filtering can effectively suppress the high-frequency image contents, and thus contribute to mitigating the negative effect of the imperfect intensity interpolator and the image noise. Specifically, Schreier et al. [26] adopted a $3 \times 3$ binomial filter on simulated noiseless speckle images and achieved a systematic error reduction in the displacements measured by DIC. Pan [29] demonstrated that the use of a $5 \times 5$ Gaussian filter prior to DIC analysis can reduce the bias error in measured displacement to a negligible degree, even though a simple bicubic interpolation is used. However, the studies about the effect of low-pass pre-filtering on DIC measurements are mainly based on the classic NR algorithm with forward additive matching strategy.

Despite its simplicity and effectiveness, there are still two unsolved problems regarding the use of the low-pass prefiltering approach in DIC/DVC analyses as pointed out in [29]. First, for the advanced DIC algorithm using inverse compositional matching strategy and iterative Gauss-Newton (i.e., IC-GN) algorithm [20], the effect of the low-pass filtering needs further in-depth investigation. In fact, although these two algorithms (i.e., FA-NR and IC-GN) are equivalent in principle, they are different in the implementation process. The IC-GN algorithm only needs to interpolate the gray values at subpixel locations, which is different from the FANR algorithm needing to interpolate gray values and gray value gradients at subpixel locations. For instance, $\mathrm{Su}$ et al. [33] found that the relative difference of systematic error between the FA-NR algorithm and the IC-GN algorithm can exceed $80 \%$. Thus, the results applicable to the FA-NR algorithm may not be simply extended to the IC-GN algorithm. Second, concerning the DVC for 3D internal displacement measurement, the iterative algorithm using tricubic interpolation gives rise to periodically subvoxel position-dependent bias error [19], [34]. Particularly, volumetric images generated by many $3 \mathrm{D}$ imaging techniques (e.g., X-ray CT scanner) have miscellaneous and higher noise levels than optical images captured by digital cameras [35]. Therefore, attention should be paid to the effect of low-pass pre-filtering on DVC when using different subvoxel registration algorithms (i.e., 3D FA-NR algorithm and 3D ICGN algorithm).

To address these two issues, we investigate the effect of low-pass pre-filtering on the 3D FA-NR algorithm and the 3D IC-GN algorithm used. In the following, we first provide a brief introduction of the two algorithms and point out their differences in the calculation process and noise robustness performance. Then, the influence of noise on the measurement accuracy of the two algorithms is analyzed.
Finally, we study the effectiveness of low-pass pre-filtering on these two subvoxel registration algorithms using simulated image sets.

\section{BASIC PRINCIPLES AND THEORETICAL ERROR ANALYSES OF TWO SUBVOXEL REGISTRATION ALGORITHMS}

\subsection{Brief introduction of two subvoxel registration algorithms}

Fig.1. shows the schematics of the 3D FA-NR algorithm and the 3D IC-GN algorithm, which both aim to optimize the zero-mean normalized sum of square difference (ZNSSD) criterion but with different matching strategies. As shown, although it has been proved by Baker and Mattews [36] that these two algorithms are equivalent in principle, the Hessian matrix (i.e., $\mathrm{H}_{12 \times 12}$ ) in the $3 \mathrm{D}$ FA-NR algorithm has to be recalculated in each iteration, while the Hessian matrix in the 3D IC-GN algorithm remains constant (does not need to be recalculated) during the iteration. Therefore, compared with the 3D FA-NR algorithm, the 3D IC-GN algorithm can achieve a faster calculation speed. Besides, there appear two other main differences during the practical implementations of these two algorithms. The first difference is the mapping strategy, the 3D FA-NR algorithm employs a forward matching strategy, in which the incremental deformation vector $\Delta \mathbf{p}$ of target subvolume obtained by optimization is directly added to the current deformation vector $\mathbf{p}$ of target subvolume to obtain the new deformation vector $\mathbf{p}$.

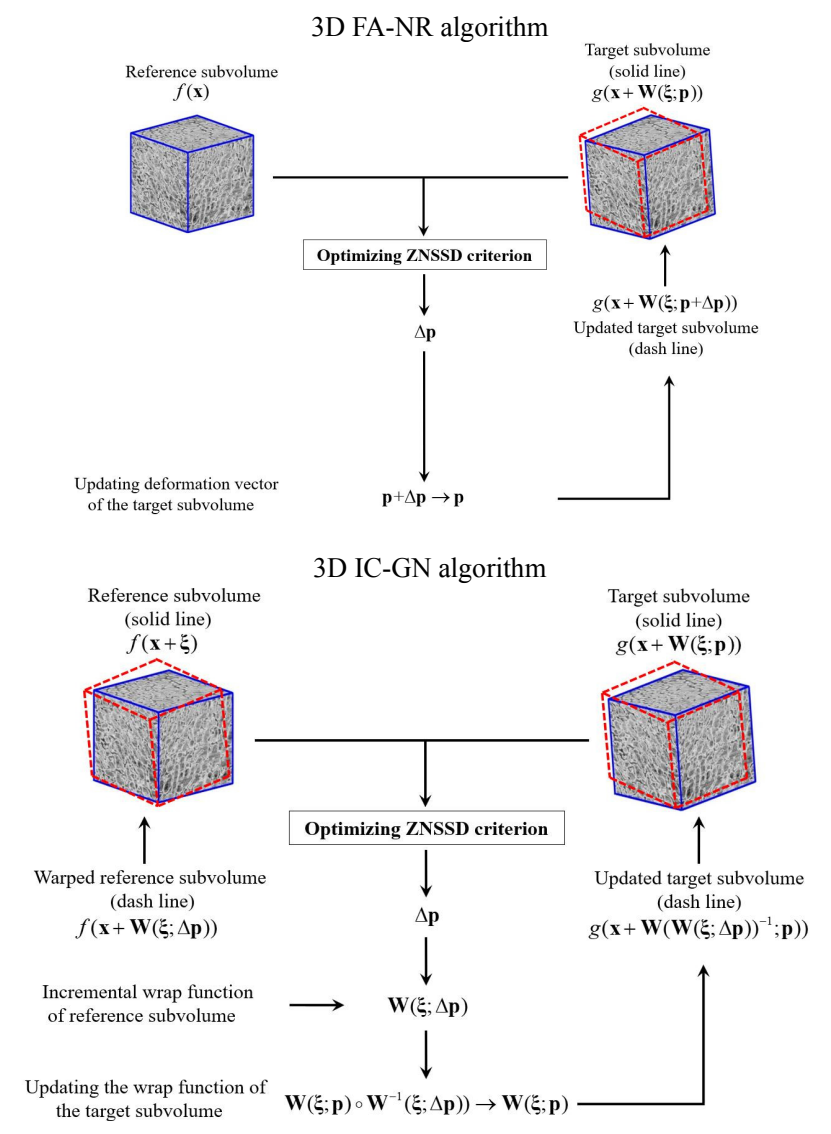

Fig.1. Schematic flow charts of the 3D FA-NR algorithm and the 3D IC-GN algorithm. 
By comparison, the 3D IC-GN algorithm adopts an inverse mapping strategy, where the incremental affine warp function $\mathbf{W}(\xi ; \Delta \mathbf{p})$ of reference subvolume obtained by optimization is inverted and composed with the current warp function $\mathbf{W}(\xi ; \mathbf{p})$ of target subvolume to obtain the new warp function $\mathbf{W}(\xi ; \mathbf{p})$. The other difference is the way to calculate the intensities and intensity gradients at subvoxel locations. In the 3D FA-NR algorithm, both the intensities $g(\mathbf{x})$ and intensity gradients $\nabla g(\mathbf{x})$ at subvoxel locations need to be provided by an interpolation method. By contrast, in the 3D IC-GN algorithm, only the intensity $g(\mathbf{x})$ at subvoxel locations must be determined by an interpolation approach and the intensity gradients $\nabla f(\mathbf{x})$ at the integer voxel location can be calculated by using the relatively simple and accurate Barron gradient operator [15].

\subsection{Theoretical error analysis of two algorithms}

In real experiments, the (volume) image inevitably contains random noise. Therefore, in the DIC/DVC analysis, the subpixel/subvoxel registration algorithm should have good noise resistibility to ensure the measurement accuracy of the displacement. Since Pan et al. [22] have given the detailed theoretical error analyses of the two algorithms in DIC, we will not repeat it in the DVC and only give the results of [22] for short. Table 1. lists the expectation and variance of the displacement measurement error $u_{e}$ of the two algorithms in the case of translation along the $x$-axis. $f_{x}$ and $g_{x}$ are the intensity gradients of noiseless reference subvolume and target subvolume, $g_{x}^{\prime}$ is the intensity gradient of noise target subvolume, $\sigma$ is the standard deviation of the white Gaussian noise of reference and deformed images, $W$ is the weight function at certain interpolation position, $h$ is the gray interpolation error.

Table 1. Theoretical results of measured displacements [22].

\begin{tabular}{|c|c|}
\hline Parameters & FA-NR algorithm \\
\hline Expectation & $\begin{array}{c}E\left(u_{e}\right)=E_{\text {int }}\left(u_{e}\right)+E_{\text {noise }}\left(u_{e}\right) \\
\cong \frac{\sum \sum g_{x} \cdot h}{\sum \sum\left(g_{x}^{\prime}\right)^{2}}+\frac{\sigma^{2} \sum \sum W}{\sum \sum\left(g_{x}^{\prime}\right)^{2}}\end{array}$ \\
\hline Variance & $\operatorname{Var}\left(u_{e}\right) \cong \frac{2 \sigma^{2}}{\sum \sum\left(g_{x}^{\prime}\right)^{2}}$ \\
\hline Parameters & IC-GN algorithm \\
\hline Expectation & $E\left(u_{e}\right)=E_{\text {int }}\left(u_{e}\right) \cong \frac{\sum \sum f_{x} \cdot h}{\sum \sum\left(f_{x}\right)^{2}}$ \\
\hline Variance & $\operatorname{Var}\left(u_{e}\right) \cong \frac{2 \sigma^{2}}{\sum \sum\left(f_{x}\right)^{2}}$ \\
\hline
\end{tabular}

It is seen that the systematic error of the FA-NR algorithm is a sum of two bias terms: interpolation-induced bias error $\left(\frac{\sum \sum g_{x} \cdot h}{\sum \sum\left(g_{x}^{\prime}\right)^{2}}\right)$ and noise-induced bias error $\left(\frac{\sigma^{2} \sum \sum W}{\sum \sum\left(g_{x}^{\prime}\right)^{2}}\right)$. By comparison, the systematic error of the IC-GN algorithm only contains one bias term: interpolation-induced bias error $\left(\frac{\sum \sum f_{x} \cdot h}{\sum \sum\left(f_{x}\right)^{2}}\right)$. The main reason is that noise-induced bias error in the IC-GN algorithm can be erased by using the Barron gray operator to calculate the intensity gradient. Meanwhile, it also can be noticed that the variances of the two algorithms are similar, namely $\left(\frac{2 \sigma^{2}}{\sum \sum\left(g_{x}^{\prime}\right)^{2}}\right.$ and $\left.\frac{2 \sigma^{2}}{\sum \sum\left(f_{x}\right)^{2}}\right)$.

\section{THE INFLUENCE OF IMAGE NOISE ON TWO SUBVOXEL REGISTRATION ALGORITHMS}

In real experiments, the measurement accuracy of DVC is influenced by many unfavorable factors. To eliminate the possible errors caused by the volume image acquisition system (e.g., self-heating effect of x-ray CT scanner [37]), imperfect loading, etc., numerical experiments are utilized in this study to analyze the influence of noise on subvoxel registration algorithm.

\subsection{Numerical translation experiments}

In the experiment, five sets of simulated volume images with precisely controlled subvoxel translation are used. The reference volume image $(200 \times 200 \times 200$ voxels, 256 gray levels) is generated using the method in [19]. Fig.2. shows the reference speckle pattern volume image and its gray distribution histogram. The deformed volume images are translated using a Fourier filter in the frequency domain [25]. Each image set contains a reference volume image and 20 deformed volume images with the subvoxel translation displacement ranging from 0.05 voxel to 1.00 voxel with the increment of 0.05 voxel in the $\mathrm{x}$-direction. The first image set is noiseless. The other four image sets are generated by adding the white Gaussian noise with zero mean and different standard deviations (varying from 1 to 4 gray value) to all 21 volume images in the first image set to simulate the image noise in real experiments.
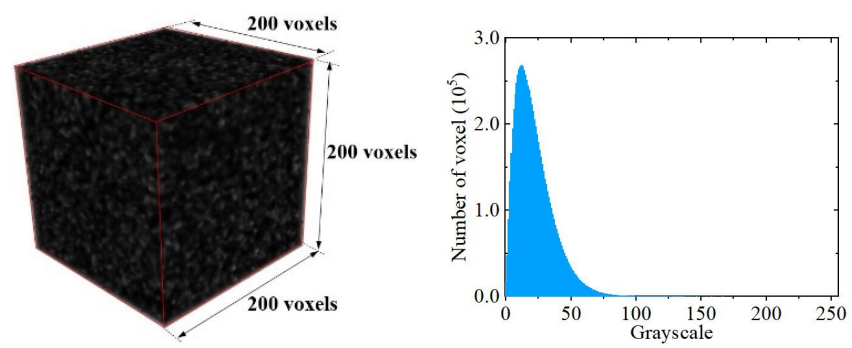

Fig.2. Reference volume image and its histogram: computersimulated 3D speckle patterns with a size of $200 \times 200 \times 200$ voxels, consisting of 96000 speckle granules.

The displacements are computed at regularly distributed $35937(=33 \times 33 \times 33)$ points using the $3 \mathrm{D} F A-N R$ algorithm $/ 3 \mathrm{D}$ IC-GN algorithm with the ZNSSD correlation criterion and zero-order shape function. The subvolume size and step size 
are $21 \times 21 \times 21$ voxels and 5 voxels, respectively. To quantitatively assess the measured displacements, the mean bias error $e_{\mathrm{u}}$ (i.e., systematic error) and standard deviation error (SD) $\sigma_{\mathrm{u}}$ (i.e., random error) are chosen. Meanwhile, although the low-pass pre-filtering for the image reduces the systematic error, it also increases the random error of the displacement measurement results. To evaluate the effect of low-pass pre-filtering more comprehensively, the root mean square error (RMSE) is adopted to characterize the total error of measurement.

$$
\left\{\begin{array}{l}
e_{u}=\bar{u}-u_{\mathrm{imp}} \\
\sigma_{u}=\sqrt{\frac{1}{N-1} \sum_{i=1}^{N}\left(u_{i}-\bar{u}\right)^{2}} \\
\operatorname{RMSE}=\sqrt{e_{u}^{2}+\sigma_{u}^{2}}
\end{array}\right.
$$

where $\bar{u}=\frac{1}{N} \sum_{i=1}^{N} u_{i}$ is the mean value of the $N(=35937)$ measured displacements and $u_{\mathrm{imp}}$ represents the imposed subvoxel displacement.

\subsection{The influence of image noise}

Fig.3. shows the mean bias errors and SD errors as a function of the actual pre-imposed subvoxel displacement of two algorithms for noiseless images (i.e., first image set). It is observed that: (1) the mean bias errors of the two algorithms show sinusoidal fluctuations with a period of 1 voxel, which attributes to the interpolation-induced error [26]; (2) the amplitude of mean bias error in the 3D IC-GN algorithm is slightly smaller than in the 3D FA-NR algorithm. Specifically, the maximum absolute value of mean bias error in the $3 \mathrm{D}$ IC-GN algorithm is $8.75 \times 10^{-4}$ voxel, while the maximum absolute value in the $3 \mathrm{D}$ FA-NR algorithm is $1.27 \times 10^{-3}$ voxel; (3) the SD error curve of the 3D IC-GN algorithm almost coincides with that of the 3D FA-NR algorithm, and their maximum values are $4.92 \times 10^{-4}$ voxel. Through the above analysis, we can get the following conclusion: when the volume images are noiseless, the systematic error difference of two algorithms is relatively small, and the two algorithms almost produce the same random error.

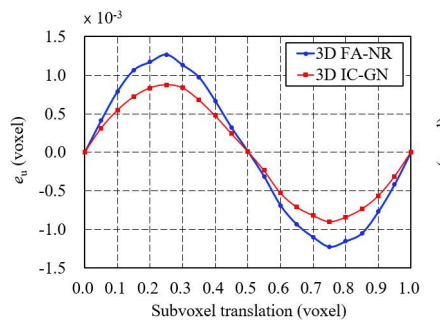

Subvoxel translation (voxel)

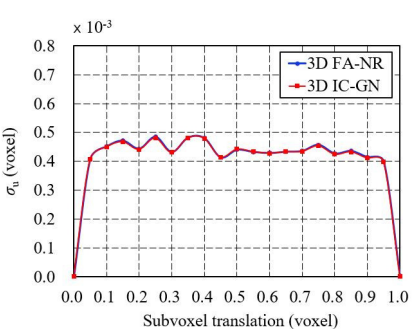

Subvoxel translation (voxel)
Fig.3. Displacement errors of the 3D FA-NR algorithm and the 3D IC-GN algorithm for noiseless volume images: (left) mean bias error, (right) SD error.
Fig.4. gives the measurement results of two algorithms under different levels of white Gaussian noise. In this situation, the systematic error includes two aspects: interpolation-induced error and noise-induced bias error. From Fig.4., it is seen that: (1) the mean bias error of the 3D FA-NR algorithm increases sharply with the increase of noise, for instance, when the standard deviation of added noise arises from 1 gray value to 4 gray value, the mean bias error has risen almost tenfold; (2) when the different levels of noise are added to the images, the mean bias error of the 3D IC-GN algorithm changes within a narrow range; (3) with the increase of noise level, the SD errors of the two algorithms also increase, for instance, when the added noise level increases from 1 gray value to 2 gray value, the SD error of the $3 \mathrm{D}$ IC-GN algorithm at 0.25 voxel displacement increases from $1.8 \times 10^{-3}$ voxel to $3.2 \times 10^{-3}$ voxel; (4) under the same noise, the mean bias error and SD error of the 3D IC-GN algorithm are smaller than the 3D FA-NR algorithm, and the more noise, the more difference.
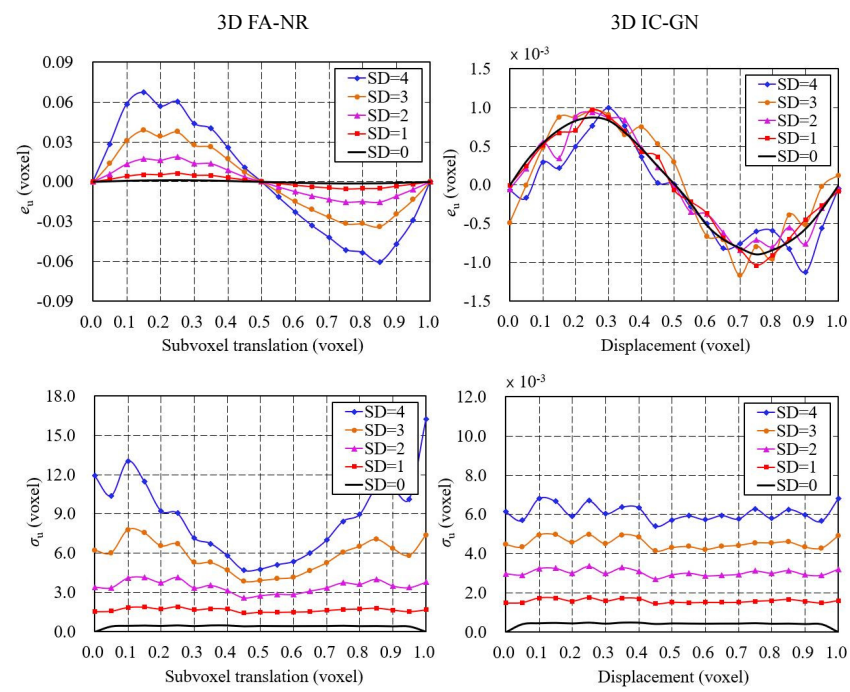

Fig.4. Displacement errors for image sets with various noise levels: (left) 3D FA-NR algorithm, (right) 3D IC-GN algorithm.

In summary, it can be concluded that the noise has little influence on the measurement accuracy of the 3D IC-GN algorithm, while it has a significant effect on the 3D FA-NR algorithm. The SD errors of the two algorithms both increase with noise, which is consistent with the existing theoretical research results [22].

\section{THE EFFECTIVENESS ANALYSIS OF LOW-PASS PRE- FILTERING ON TWO SUBVOXEL REGISTRATION ALGORITHMS}

Related researches indicate that prior to the DIC analysis, the low-pass pre-filtering can effectively reduce noise influence. Reference [26] showed that applying a binomial filter $[0.25,0.5,0.25]$ prior to correlation can effectively reduce the bias error caused by interpolation in the Levenberg-Marquardt algorithm. The effectiveness of the Gaussian low-pass filter on the interpolation error reduction of the FA-NR algorithm was verified in [29]. In this section, the effectiveness of these two filters on the 3D FA-NR 
algorithm and the 3D IC-GN algorithm is testified, respectively.

During the volume image pre-filtering, the 3D kernel can be implemented by three successive one-dimensional (1D) convolutions in $\mathrm{x}, \mathrm{y}$, and $\mathrm{z}$ direction. The $1 \mathrm{D}$ kernels of the binomial filter with different sizes are listed in Table 2 .

Table 2. 1D kernel of the binomial filter with different sizes.

\begin{tabular}{|c|c|}
\hline Size & Binomial filter \\
\hline $3 \times 3 \times 3$ voxel & $1 / 4\left[\begin{array}{lll}1 & 2 & 1\end{array}\right]$ \\
\hline $5 \times 5 \times 5$ voxel & 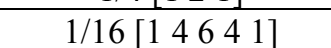 \\
\hline $7 \times 7 \times 7$ voxel & $1 / 64\left[\begin{array}{lllllll}1 & 6 & 15 & 20 & 15 & 6 & 1\end{array}\right]$ \\
\hline
\end{tabular}

Meanwhile, the 1D kernel of a Gaussian filter is obtained by

$$
g(x)=C e^{-x^{2} / 2 \sigma^{2}}
$$

where $x$ is the distance from the origin and ranges from $-m$ to $m$. The kernel size is $2 m+1 . \sigma$ is the SD of the Gaussian function. In this work, the size of the Gaussian filter (denoted as $h)$ is chosen to be $h=(4 \sigma+1) \times(4 \sigma+1)$. Factor $C$ is chosen to ensure the kernel sums up to unity.

\subsection{Subvoxel translation}

Two $5 \times 5 \times 5$ filters are adopted to filter all volume images of two image sets generated in Section 3.1: the first image set without noise and the fifth image set which has a 4 gray value noise, respectively. The $u$ displacement results of two image sets in three situations (i.e., without filter, the volume images are filtered by the binomial filter, and the volume images are filtered by Gaussian filter) of the two algorithms are shown in Fig.5. and Fig.6., respectively. From Fig.5., it can be seen that the results of the two filters are similar. When using the 3D FA-NR algorithm, the mean bias error for noiseless images almost remains unchanged before and after prefiltering, while the SD error has a significant increase. On the other hand, when using the 3D IC-GN algorithm, the mean bias error shows a slight decrease after pre-filtering. Like the 3D FA-NR algorithm, the SD error of the 3D IC-GN algorithm also increases significantly. From the results of the total error of the measurement results, the low-pass prefiltering of the noiseless image will lead to the increase of the total error of the displacement measurement results, but the increase is not significant.

For noise volume images, from Fig.6., it can be seen that in the 3D FA-NR algorithm no matter which filter is used, the mean bias error is lowered greatly, especially after prefiltering, the maximum mean bias error declines to $97 \%$. Meanwhile, in the 3D IC-GN algorithm, the amplitude of the sinusoidal error curve is also decreased after pre-filtering. The peak value of the curve has more than halved. For instance, at 0.25 voxel displacement, before pre-filtering, the mean bias error is $8.8 \times 10^{-4}$ voxel. It becomes $3.1 \times 10^{-4}$ voxel and $2.9 \times 10^{-4}$ voxel when using the binomial filter and Gaussian filter, respectively. However, it is obvious that the effectiveness of pre-filtering on the 3D FA-NR algorithm is more distinct than on the 3D IC-GN algorithm.
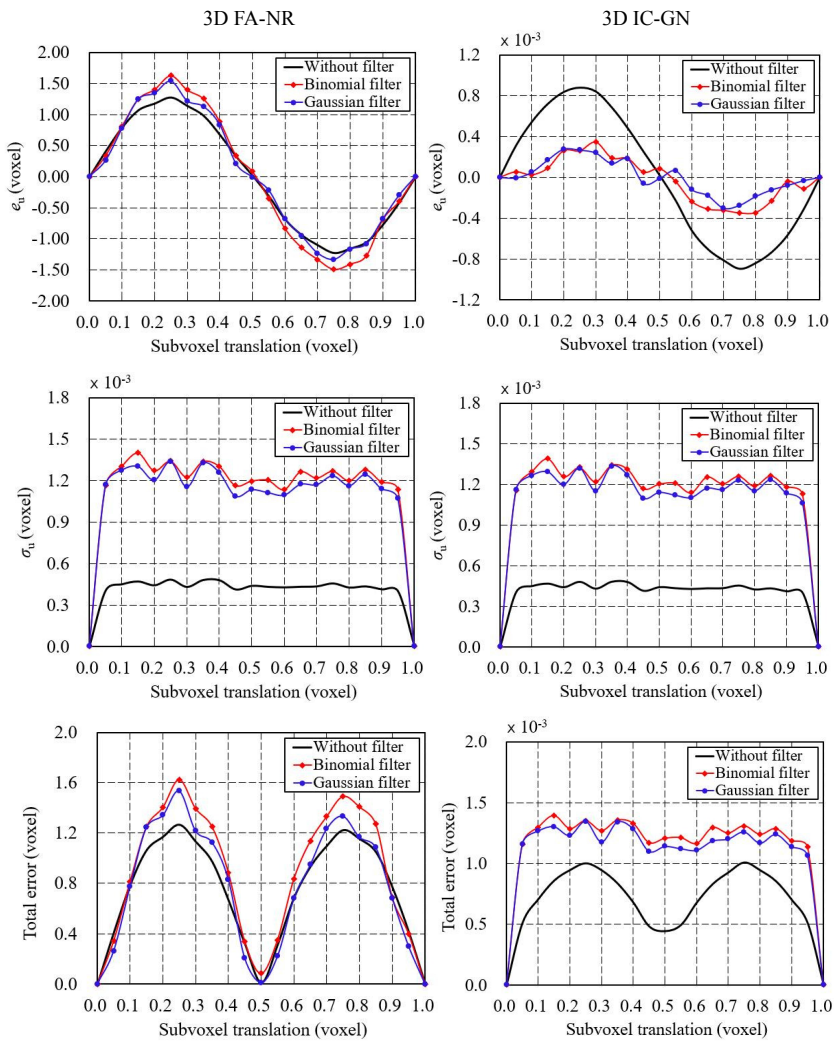

Fig.5. Displacement errors for noiseless volume images using different filters: (left) 3D FA-NR algorithm, (right) 3D IC-GN algorithm.
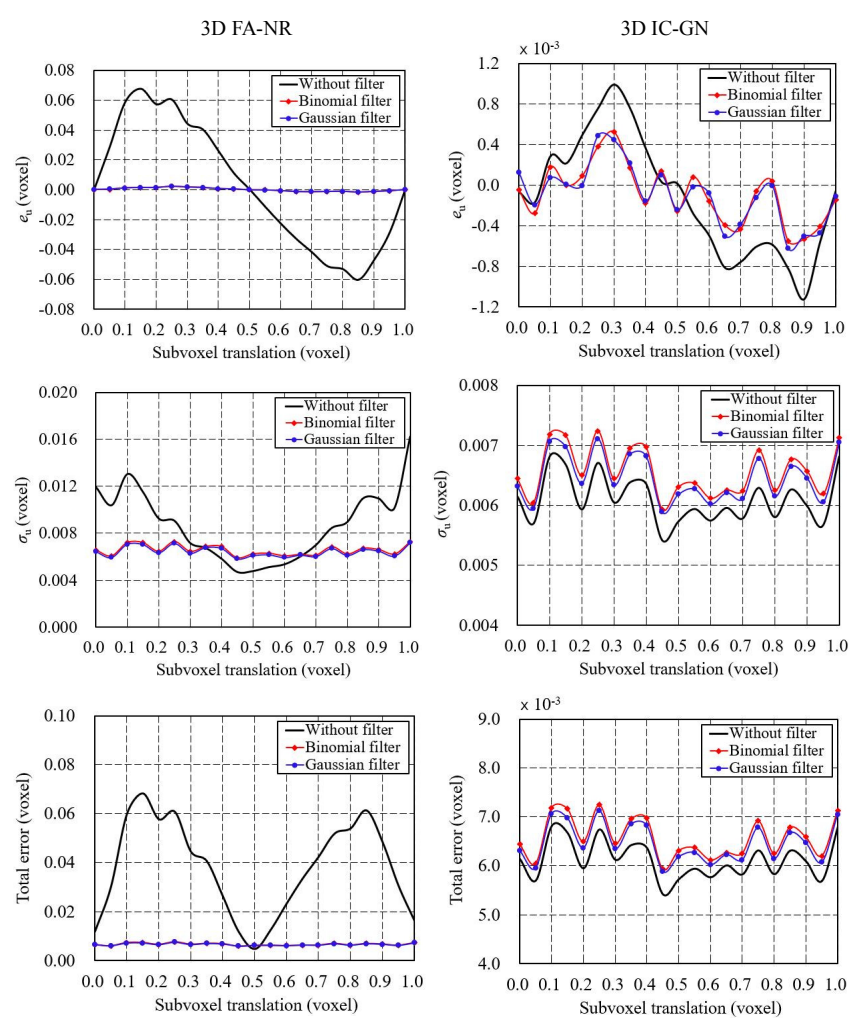

Fig.6. Displacement errors for noise volume images (noise, $\mathrm{SD}=4$ ) using different filters: (left) 3D FA-NR algorithm, (right) 3D IC-GN algorithm. 
On the other hand, it can also be seen from Fig.6. that after pre-filtering there is no significant change of the SD error of the 3D FA-NR algorithm. However, in the 3D IC-GN algorithm, it can be noticed that using low-pass pre-filtering can result in an increase of the SD error. The reason for the increase of the SD error is that pre-filtering images will lessen the sum of square of subset intensity gradients (SSSIG) [38] value in the subvolume, and the SSSIG value is inverse proportional to the SD error of a subvolume in the IC-GN algorithm. Therefore, pre-filtering images will cause an increase in the SD error.

\subsection{Complex deformation}

To further testify the effectiveness of low-pass pre-filtering, we use the numerical indentation experiment to test the performance of low-pass pre-filtering in a more complex deformation situation. Meanwhile, through the above analysis, we can find that the difference between a binomial filter and a Gaussian filter is small, so we just use the Gaussian filter in this section.

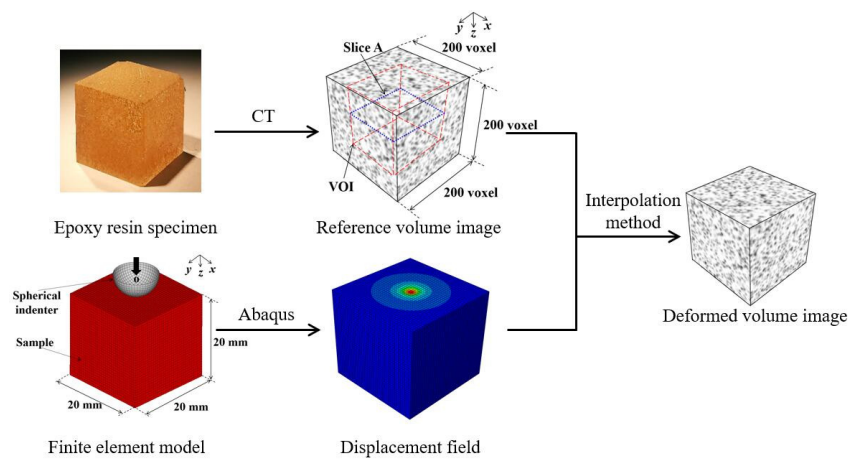

Fig.7. Synthesizing the deformed volume image.

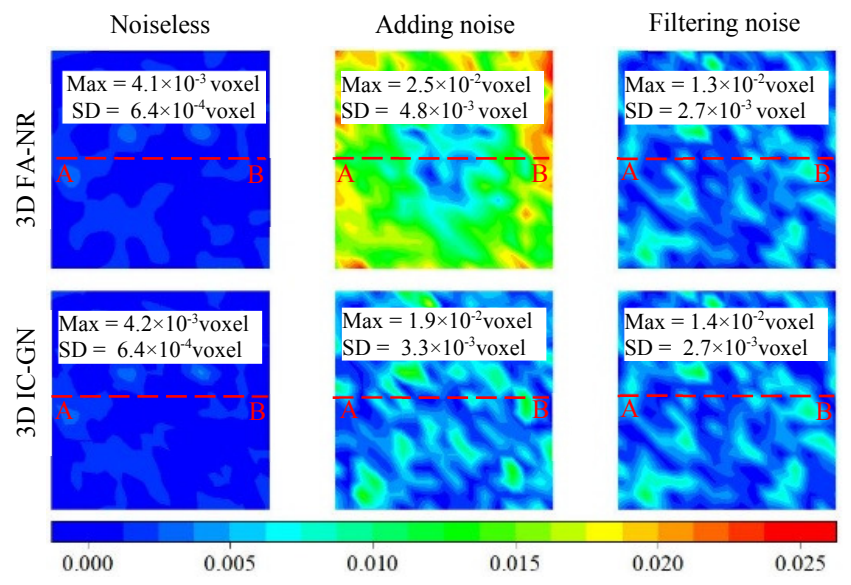

Fig.8. Comparison of w displacement error of two algorithms on slice $\mathrm{A}$.

The volume image $(200 \times 200 \times 200$ voxels $)$ obtained by micro-CT of epoxy resin composite embedded copper particles is adopted as the reference volume image. The deformed volume image is synthesized using the interpolation method based on the reference volume image, as shown in Fig.7. First, the numerical analysis of the indentation experiment is carried out in Abaqus to obtain the displacement field. Then, using the image interpolation method [39], the obtained displacement field can be added to the reference volume image to synthesize the deformed volume image. Specifically, the obtained displacement is interpolated to the integer voxel positions of the image and the reference volume image is deformed according to this interpolated field. This will result in a grey value map at noninteger voxel positions. Accordingly, the grey values at the integer voxel positions are obtained by interpolation of the non-integer ones. This is the deformed volume image. In the finite element model, the specimen size is set to be $20 \times 20 \times 20 \mathrm{~mm}$, and the radius of the indenter is $2.5 \mathrm{~mm}$. The indenter is considered as a rigid body and the specimen is taken as a linear elastic body, whose elastic modulus and Poisson's ratio are $2.2 \mathrm{GPa}$ and 0.2 , respectively. The bottom end of the specimen is constrained in all degrees of freedom and the indenter only has the translational degree of freedom in the z-direction. The displacement of the indenter is $0.3 \mathrm{~mm}$. The contact between the specimen and the indenter is assumed to be frictionless.
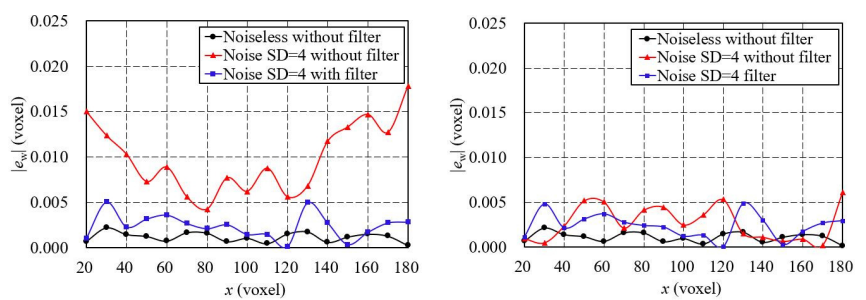

Fig.9. Comparison of w displacement error between two algorithms along the line $\mathrm{AB}$ in three situations: (left) 3D FA-NR algorithm, (right) 3D IC-GN algorithm.

Table 3. Statistics of all calculation points in VOI between two algorithms.

\begin{tabular}{|c|c|c|c|}
\hline \multirow[b]{2}{*}{$\begin{array}{c}\text { Measurement } \\
\text { error }\end{array}$} & \multicolumn{3}{|c|}{ 3D FA-NR algorithm } \\
\hline & $\begin{array}{c}\text { Noiseless } \\
\text { without } \\
\text { filter }\end{array}$ & $\begin{array}{c}\text { Noise } \\
\mathrm{SD}=4 \\
\text { without } \\
\text { filter }\end{array}$ & $\begin{array}{c}\text { Noise } \\
\mathrm{SD}=4 \\
\text { with filter }\end{array}$ \\
\hline $\begin{array}{l}\text { Mean abs } \\
\text { error }(\mathrm{mm})\end{array}$ & $9.0 \times 10^{-4}$ & $1.8 \times 10^{-2}$ & $3.3 \times 10^{-3}$ \\
\hline SD error $(\mathrm{mm})$ & $6.0 \times 10^{-4}$ & $7.7 \times 10^{-3}$ & $2.4 \times 10^{-3}$ \\
\hline $\begin{array}{l}\text { Total error } \\
(\mathrm{mm})\end{array}$ & $1.1 \times 10^{-3}$ & $2.0 \times 10^{-2}$ & $4.1 \times 10^{-3}$ \\
\hline \multirow[b]{2}{*}{$\begin{array}{c}\text { Measurement } \\
\text { error }\end{array}$} & \multicolumn{3}{|c|}{ 3D IC-GN algorithm } \\
\hline & $\begin{array}{c}\text { Noiseless } \\
\text { without } \\
\text { filter }\end{array}$ & $\begin{array}{c}\text { Noise } \\
\mathrm{SD}=4 \\
\text { without } \\
\text { filter }\end{array}$ & $\begin{array}{c}\text { Noise } \\
\mathrm{SD}=4 \\
\text { with filter }\end{array}$ \\
\hline $\begin{array}{l}\text { Mean abs } \\
\text { error ( } \mathrm{mm})\end{array}$ & $9.0 \times 10^{-4}$ & $3.8 \times 10^{-3}$ & $3.3 \times 10^{-3}$ \\
\hline SD error $(\mathrm{mm})$ & $5.0 \times 10^{-4}$ & $2.9 \times 10^{-3}$ & $2.4 \times 10^{-3}$ \\
\hline $\begin{array}{l}\text { Total error } \\
(\mathrm{mm})\end{array}$ & $1.0 \times 10^{-3}$ & $4.8 \times 10^{-3}$ & $4.1 \times 10^{-3}$ \\
\hline
\end{tabular}

In the DVC analysis, the subvolume and step size are set to be $21 \times 21 \times 21$ voxels and 5 voxels, respectively. There are 
$33 \times 33 \times 33=35937$ regularly distributed calculation points. In the test, $w$ displacement measurement error (the absolute value of the difference between the imposed $w$ displacement and the measured one) is used to assess the measurement accuracy. Fig.8. shows the $w$ displacement error of the two algorithms on slice A ( $\mathrm{z}=90$ voxel, shown in Fig.7.) in three situations, including noiseless volume image, noise volume image (adding $\mathrm{SD}=4$ white Gaussian noise to the noiseless volume image) and noise volume image filtered by $5 \times 5 \times 5$ Gaussian filter. It can be seen from Fig.8. that if the images are not filtered by the Gaussian filter, the displacement error of the 3D FA-NR algorithm is large. After using the Gaussian filter, the error will decrease greatly. For the 3D IC-GN algorithm, the displacement error has no obvious change before and after pre-filtering.

Detailed displacement error comparison between the two algorithms along the line $\mathrm{AB}$ on slice $\mathrm{A}(\mathrm{y}=90$ voxel, shown in Fig.8.) is shown in Fig.9. It is seen that in the 3D FA-NR algorithm, after adding the white Gaussian noise, the increase of the displacement error is obvious. If the image is filtered by the Gaussian filter, the error is back to where it was before adding noise. On the contrary, the results of the 3D IC-GN algorithm stay at the same level in all three situations. Table 3. lists the statistics of all calculation points, including mean displacement error, SD error, and total error. From the figure, it can also be seen that the low-pass pre-filtering is very useful in the 3D FA-NR algorithm but has no obvious effect on the 3D IC-GN algorithm.

\section{CONCLUSION}

In this work, the effects of low-pass pre-filtering on DVC when using two mainstream subvoxel registration algorithms (3D FA-NR algorithm and 3D IC-GN algorithm) are investigated. The influences of image noise on the accuracy and precision of the two subvoxel registration algorithms are first analyzed by numerical translation experiments. Then the effects of the low-pass pre-filtering approach on the two algorithms are examined using simulated images with different noise levels and deformation modes. This work clearly addressed the unsolved problems posed in our previous work [29] regarding the effects of low-pass prefiltering on the DIC/DVC algorithms. Valuable conclusions are summarized as follows:

1) Random image noise has little influence on the systematic error of the 3D IC-GN algorithm but has a great influence on the 3D FA-NR algorithm. When the images are noiseless, the systematic error and random error of the two algorithms are almost the same. However, with the increase of noise, the systematic error of the 3D FA-NR algorithm increases sharply, while the 3D IC-GN algorithm only fluctuates slightly. The random errors of the two algorithms are the same and increase with noise.

2) Low-pass pre-filtering can effectively improve the accuracy of the 3D FA-NR algorithm but the effect of lowpass pre-filtering on 3D IC-GN algorithm is not so obvious as on 3D FA-NR algorithm. Since real experimental images unavoidably contain certain noise, the 3D IC-GN algorithm, which can effectively diminish the influence of image noise on measured displacement, is highly recommended for practical use. However, as the measurement accuracy of the 3D FA-NR algorithm is greatly affected by image noise, if using it in DVC, low-pass pre-filtering must be used to reduce the impact of image noise, particularly a low-order interpolation algorithm is used.

It should be pointed out that the results of this study are obtained from numerical experiments, and only Gaussian noise is considered. However, as real experimental volume images may be contaminated by many factors such as thermal noise, readout noise, etc., only computer-simulated speckle volume images were used in this research. Whether the results can be effectively applied to real volumetric images contaminated with a variety of complicated noise still requires further research.

\section{ACKNOWLEDGMENT}

This work is supported by the National Natural Science Foundation of China (Grant nos. 11925202, 11872009, and 11632010) and the Academic Excellence Foundation of BUAA for PhD Students.

\section{REFERENCES}

[1] Bay, B.K., Smith, T.S., Fyhrie, D.P., Saad, M. (1999). Digital volume correlation: Three-dimensional strain mapping using X-ray tomography. Experimental Mechanics, 39 (3), 217-226.

[2] Pan, B., Wang, B. (2020). Some recent advances in digital volume correlation. Optics \& Lasers in Engineering, https://doi.org/10.1016/j.optlaseng.2020. 106189.

[3] Bay, B.K. (2008). Methods and applications of digital volume correlation. The Journal of Strain Analysis for Engineering Design, 43 (8), 745-760.

[4] Roux, S., Hild, F., Viot, P., Bernard, D. (2008). Threedimensional image correlation from X-ray computed tomography of solid foam. Composites Part A: Applied Science \& Manufacturing, 39 (8), 1253-1265.

[5] Benoit, A., Guérard, S., Gillet, B., et al. (2009). 3D analysis from micro-MRI during in situ compression on cancellous bone. Journal of Biomechanics, 42 (14), 2381-2386.

[6] Poinard, C., Piotrowska, E., Malecot, Y., Daudeville, L., Landis, E.N. (2012). Compression triaxial behavior of concrete: The role of the mesostructure by analysis of X-ray tomographic images. European Journal of Environmental and Civil Engineering, 16, 115-136.

[7] Mao, L., Yuan, Z., Yang M., Liu, H., Chiang, F. (2019). 3D strain evolution in concrete using in situ X-ray computed tomography testing and digital volumetric speckle photography. Measurement, 133, 456-467.

[8] Wang, B., Pan, B., Lubineau, G. (2018). Morphological evolution and internal strain mapping of pomelo peel using X-ray computed tomography and digital volume correlation. Materials \& Design, 137, 305-315.

[9] Mendoza, A., Schneider, J., Parra, E., Obert, E., Roux, S. (2019). Differentiating 3D textile composites: A novel field of application for Digital Volume Correlation. Composite Structures, 208, 735-743.

[10] Shahbazi, M. (2013). Hybrid 3D dynamic measurement by particle swarm optimization and photogrammetric tracking. Measurement Science Review, 13 (6), 298304. 
[11] Pan, B. (2018). Digital image correlation for surface deformation measurement: Historical developments, recent advances and future goals. Measurement Science and Technology, 29 (8), 082001.

[12] Bruck, H.A., McNeill, S.R., Sutton, M.A., Peters III, W.H. (1989). Digital image correlation using NewtonRaphson method of partial differential correction. Experimental Mechanics, 29 (3), 261-267.

[13] Zhang, J., Jin, G., Ma, S., Meng, L. (2003). Application of an improved subpixel registration algorithm on digital speckle correlation measurement. Optics \& Laser Technology, 35 (7), 533-542.

[14] Hung, P.C., Voloshin, A.S. (2003). In-plane strain measurement by digital image correlation. Journal of the Brazilian Society of Mechanical Sciences and Engineering, 25 (3), 215-221.

[15] Pan, B., Xie, H., Xu, B., Dai, F. (2006). Performance of sub-pixel registration algorithms in digital image correlation. Measurement Science and Technology, 17 (6), 1615.

[16] Pan, B., Asundi, A., Xie, H., Gao, J. (2009). Digital image correlation using iterative least squares and pointwise least squares for displacement field and strain field measurements. Optics \& Lasers in Engineering, 47, 865-874.

[17] Pan, B., Li, K. (2011). A fast digital image correlation method for deformation measurement. Optics \& Lasers in Engineering, 49 (7), 841-847.

[18] Huang, J., Pan, X., Li, S., Peng, X., Xiong, C., Fang, J. (2011). A digital volume correlation technique for 3-D deformation measurements of soft gels. International Journal of Applied Mechanics, 3 (2), 335-354.

[19] Pan, B., Wu, D., Wang, Z. (2012). Internal displacement and strain measurement using digital volume correlation: A least-squares framework. Measurement Science and Technology, 23 (4), 045002.

[20] Pan, B., Li, K., Tong, W. (2013). Fast, robust and accurate digital image correlation calculation without redundant computations. Experimental Mechanics, 53 (7), 1277-1289.

[21] Shao, X., Dai, X., He, X. (2015). Noise robustness and parallel computation of the inverse compositional Gauss-Newton algorithm in digital image correlation. Optics \& Lasers in Engineering, 71, 9-19.

[22] Pan, B., Wang, B. (2016). Digital image correlation with enhanced accuracy and efficiency: A comparison of two subpixel registration algorithms. Experimental Mechanics, 56 (8), 1395-1409.

[23] Jiang, Z., Kemao, Q., Miao, H., Yang, J., Tang, L. (2015). Path-independent digital image correlation with high accuracy, speed and robustness. Optics \& Lasers in Engineering, 65, 93-102.

[24] Li, W., Li, Y., Liang, J. (2019). Enhanced feature-based path-independent initial value estimation for robust point-wise digital image correlation. Optics and Lasers \& Engineering, 121, 189-202.

[25] Schreier, H.W., Sutton, M.A. (2002). Systematic errors in digital image correlation due to undermatched subset shape functions. Experimental Mechanics, 42 (3), 303310 .
[26] Schreier, H.W., Braasch, J.R., Sutton, M.A. (2000). Systematic errors in digital image correlation caused by intensity interpolation. Optical Engineering, 39 (11), 2915-2921.

[27] Tong, W. (2011). Subpixel image registration with reduced bias. Optics letters, 36 (5), 763-765.

[28] Bay, B.K., Smith, T.S., Fyhrie, D.P. (1999). Digital volume correlation: Three-dimensional strain mapping using X-ray tomography. Experimental Mechanics, 39 (3), 217-226.

[29] Pan, B. (2013). Bias error reduction of digital image correlation using Gaussian pre-filtering. Optics \& Lasers in Engineering, 51 (10), 1161-1167.

[30] Shahbazi, M. (2013). Hybrid 3D dynamic measurement by particle swarm optimization and photogrammetric tracking. Measurement Science Review, 13 (6), 298304.

[31] Wang, B., Pan, B. (2019). Self-adaptive digital volume correlation for unknown deformation fields. Experimental Mechanics, 59 (2), 149-162.

[32] Pan, B., Zou, X. (2020). Quasi-Gauss point digital image/volume correlation: A simple approach for reducing systematic errors due to undermatched shape functions. Experimental Mechanics, 1-12.

[33] Su, Y., Zhang, Q., Xu, X., Gao, Z., Wu, S. (2018). Interpolation bias for the inverse compositional GaussNewton algorithm in digital image correlation. Optics \& Lasers in Engineering, 100, 267-278.

[34] Pan, B., Wang, B., Wu, D., Lubineau, G. (2014). An efficient and accurate 3D displacements tracking strategy for digital volume correlation. Optics \& Lasers in Engineering, 58, 126-135.

[35] Limodin, N., Réthoré, J., Adrien, J., et al. (2011). Analysis and artifact correction for volume correlation measurements using tomographic images from a laboratory X-ray source. Experimental Mechanics, 51 (6), 959-970.

[36] Baker, S., Matthews, I. (2001). Equivalence and efficiency of image alignment algorithms. In 2001 IEEE Computer Society Conference on Computer Vision and Pattern Recognition (CVPR 2001). IEEE.

[37] Wu, T.Y., Lin, S.F. (2013). A method for extracting suspected parotid lesions in CT images using featurebased segmentation and active contours based on stationary wavelet transform. Measurement Science Review, 13 (5), 237-247.

[38] Pan, B., Xie, H., Wang, Z., Qian, K., Wang, Z. (2008). Study on subset size selection in digital image correlation for speckle patterns. Optics Express, 16 (10), 7037-7048.

[39] Lava, P., Cooreman, S., Coppieters, S., De Strycker, M., Debruyne, D. (2009). Assessment of measuring errors in DIC using deformation fields generated by plastic FEA. Optics \& Lasers in Engineering, 47 (7-8), $747-753$

Received March 10, 2020 Accepted September 14, 2020 